\title{
A Simple HPLC-MS/MS Method for Quantification of Naltrexone and 6-beta Naltrexol: An Application to Effect of Uremic Toxins on Metabolic Reduction
}

\author{
Osama Yousef Alshogran ${ }^{1, *}$, Aref Louai Zayed ${ }^{2}$ \\ 'Department of Clinical Pharmacy, Faculty of Pharmacy, Jordan University of Science and Technology, Irbid, JORDAN. \\ 2Department of Medicinal Chemistry and Pharmacognosy, Faculty of Pharmacy, Jordan University of Science and Technology, Irbid, JORDAN.
}

\begin{abstract}
Objective: This study aimed to 1) develop and validate a simple, accurate and reproducible high-performance liquid chromatography-tandem mass spectrometry method for determination and quantification of naltrexone and its reduced metabolite (6-beta naltrexol); 2) assess the effect of uremic toxins on metabolic reduction. Methods: Sample preparation was conducted through liquid-liquid extraction of analytes spiked in $10 \mathrm{mM}$ Tris- $\mathrm{HCl}$ incubation buffer ( $\mathrm{pH}$ 7.4) using methyl tert-butyl ether. The chromatographic separation of analytes was achieved using Inert Sustain $\mathrm{C}_{18}(2.1 \times 50 \mathrm{~mm}, 5$ $\mu \mathrm{m}$ ) analytical column under isocratic elution of solvent $A$ (water containing $0.1 \%$ formic acid) and solvent B (methanol containing $0.1 \%$ formic acid) over a run time of $5 \mathrm{~min}$. The analytes were detected by multiple reaction monitoring with electrospray ionization in the positive mode. Results: Excellent linearity was observed for all analytes over the concentration ranges of $100-8,000 \mathrm{ng} / \mathrm{mL}$ for naltrexone and $10-800 \mathrm{ng} / \mathrm{mL}$ for 6-beta naltrexol. The intra- and inter-day accuracy and precision for analytes were within $\pm 15.0 \%$. The method is accurate and precise and novel in its application
\end{abstract}

to in vitro experiments assessing the effect of uremic toxins on metabolic reduction of naltrexone, which showed that indoxyl sulfate $(100 \mu \mathrm{M})$ is a significant inhibitor of naltrexone reduction. Conclusion: The developed method is reproducible and accurate and well suited for conducting enzyme kinetic studies assessing metabolic reduction.

Key words: Naltrexone, 6-beta naltrexol, Reduction, LC-MS, Enzyme kinetics, Uremic toxins.

\section{Correspondence}

Dr. Osama Yousef Alshogran,

Assistant Professor, Department of Clinical Pharmacy, Faculty of Pharmacy, Jordan University of Science and Technology, Irbid- 22110, JORDAN.

Phone: +96227201000

Email: oyalshogran@just.edu.jo

DOI: 10.5530/jyp.2019.11.53

\section{INTRODUCTION}

Naltrexone is an opioid antagonist used in the treatment of opiate dependence and alcoholism. ${ }^{1}$ It works by binding and inhibiting of beta opioid receptors located in brain. Naltrexone is a highly metabolized drug that undergoes hepatic reduction at the carbonyl moiety to produce primarily 6-beta naltrexol metabolite., ${ }^{2,3}$ Naltrexone reduction is principally mediated by hepatic Aldo-keto Reductase (AKR) isoforms located in cytosol. ${ }^{3-5}$ While the 6-alpha metabolite has not been recognized in hu

mans, the beta isoform is pharmacologically active and inhibits opioid receptors to levels lower than that of the parent drug. Additionally, the plasma half-life of 6-beta naltrexol is longer than that of naltrexone and it is detected in plasma at higher levels, which may contribute to the clinical effects observed with naltrexone treatment. ${ }^{6}$

As naltrexone is chiefly metabolized by reduction, the drug may act as a candidate of reductase enzyme probe substrates. Therefore, naltrexone reduction may be utilized to study the effect of disease state and therapeutic treatments on metabolic reduction to gain a better understanding of this metabolic pathway. For instance, kidney disease has been recently shown to alter drug reduction in vitro. ${ }^{7}$ However, the mechanisms behind this alteration are unclear. In order to assess factors contributing to alter metabolic reduction, it is necessary to have a robust and validated analytical method suitable for the quantitative determination of 6-beta naltrexol metabolite generated from in vitro experiments.

Because of inherent selectivity and specificity of Mass Spectrometry (MS), multiple analytical methods have been developed utilizing High
Performance Liquid Chromatography (HPLC) coupled with MS detection for the determination of naltrexone, with only few assays have quantified naltrexone and 6-beta naltrexol simultaneously. ${ }^{8-13}$ Additionally, these methods were validated in biological fluids such as serum, plasma and blood of animals or human. Up to our knowledge, there were no reports specifically designed to quantify 6-beta naltrexol following in vitro enzyme kinetic studies. ${ }^{4,5}$ Thus, we developed and partially validated a simple and rapid HPLC-MS/MS assay for the simultaneous determination of naltrexone and 6-beta naltrexol metabolite following in vitro incubations. The method was used to examine, for the first time, the effect of uremic toxins on cytosolic reduction of naltrexone.

\section{MATERIALS AND METHODS}

\section{Chemicals and Reagents}

Naltrexone $\left(\mathrm{C}_{20} \mathrm{H}_{23} \mathrm{NO}_{4}\right)$ and 6-beta naltrexol $\left(\mathrm{C}_{20} \mathrm{H}_{25} \mathrm{NO}_{4}\right)$ were purchased from Toronto Research Chemicals (Canada) and Sigma (USA), respectively. Colchicine $\left(\mathrm{C}_{22} \mathrm{H}_{25} \mathrm{NO}_{6}\right.$, used as internal standard), hippuric acid, P-cresol, magnesium chloride, 3-carboxy-4-methyl-5-propyl2-furanpropanoic acid (CMPF) and testosterone were purchased from Acros (USA). Tris (hydroxymethyl) aminomethane (Trizma base) was obtained from Bio Basic (Canada). Creatinine, indole-3-acetic acid and NADPH were purchased from Combi-Blocks Inc (USA). Indoxyl sulfate potassium salt was purchased from Santa Cruz Biotechnology (USA). Chemical solvents were purchased from Thermo Fisher Scientific (USA) 
or BBC Chemicals (USA). All chemicals were with the highest purity and analytical grade available. Nitrogen gas (ultra-pure, $>99.9 \%$ ) was produced by a Parker nitrogen generator (USA). Pooled human liver cytosol $(20 \mathrm{mg} / \mathrm{mL})$ was obtained from Gibco/ThermoFisher Scientific (USA).

\section{Equipment and HPLC-MS/MS Conditions}

Liquid chromatography was performed with an Agilent 1200 Series HPLC system (Agilent Technologies, CA, USA) including an autosampler and high-performance binary pump. Chromatographic separation of the samples was achieved with Inert Sustain $C_{18}(2.1 \times 50 \mathrm{~mm}, 5 \mu \mathrm{m}$, GL Sciences, Japan) analytical column. Analytes were separated under isocratic mobile phase over flow rate of $200 \mu \mathrm{L} / \mathrm{min}$ and composed of $20 \%$ solvent A (water containing $0.1 \%$ formic acid) and $80 \%$ solvent $\mathrm{B}$ (methanol containing $0.1 \%$ formic acid). The total run time was $5 \mathrm{~min}$. The autosampler was maintained at $10^{\circ} \mathrm{C}$ and the column temperature was held at $40^{\circ} \mathrm{C}$.

MS/MS detection was performed on API- 3200 triple quadruple mass spectrometer (Applied Biosystems/ MDS Sciex) equipped with a heated electrospray ionization source. Analytes were detected in positive ionization mode using multiple reaction monitoring with a dwell time of 150 $\mathrm{ms}$. The spray voltage was set to $5500 \mathrm{~V}$ and the ion source temperature was set to $450^{\circ} \mathrm{C}$. The sheath gas and auxiliary gas were set to 45 and 50 (arbitrary units), respectively. Collision gas pressure was set at 5 mTorr. The ion transitions were $m / z 342.2 \rightarrow 322.8$ for naltrexone (collision energy $=27 \mathrm{~V}$ ), $m / z 344.2 \rightarrow 324.8$ for 6-beta naltrexol (collision energy $=$ $25 \mathrm{~V}$ ) and $\mathrm{m} / z 400.2 \rightarrow 152.1$ for the internal standard colchicine (collision energy $=115 \mathrm{~V}$ ). Data were processed with Analyst Software (version 1.6.2, Applied Biosystems/ SCIEX).

\section{Preparation of Calibration Standards and Quality Controls}

Naltrexone was dissolved in methanol and 6-beta naltrexol was dissolved in DMSO to prepare 1.0 and $5.0 \mathrm{mg} / \mathrm{mL}$ stock solutions, respectively. These solutions were spiked into $10 \mathrm{mM}$ Tris- $\mathrm{HCl}$ incubation buffer $(\mathrm{pH}$ 7.4) to create working solutions that were used to prepare calibration standards at concentrations of 100,150, 300, 600, 1500, 3000, 6000 and $8000 \mathrm{ng} / \mathrm{mL}$ for naltrexone and 10, 15, 30, 60, 150, 300, 600 and $800 \mathrm{ng} /$ $\mathrm{mL}$ for 6-beta naltrexol. Three quality control (QC) samples (LQC, MQC and HQC) containing both analytes were made by spiking analyte stock and working solutions into Tris- $\mathrm{HCl}$ buffer to prepare three concentration levels of 400, 2000 and $7000 \mathrm{ng} / \mathrm{mL}$ for naltrexone and 40, 200 and $700 \mathrm{ng} / \mathrm{mL}$ for 6-beta naltrexol metabolite. Stock solutions were stored at $-40^{\circ} \mathrm{C}$, whereas calibration standards and QC samples were stored at $4{ }^{\circ} \mathrm{C}$.

\section{Sample Preparation}

The internal standard colchicine was initially prepared in methanol at stock concentration of $1.0 \mathrm{mg} / \mathrm{L}$ that was further diluted in Tris- $\mathrm{HCl}$ buffer to prepare a working solution containing $100 \mu \mathrm{g} / \mathrm{mL}$. A $200 \mu \mathrm{L}$ aliquot of sample (calibration standards, QCs, blanks, application samples) was spiked with $20 \mu \mathrm{L}$ internal standard solution and vortexed. Liquidliquid extraction was carried out by adding $2 \mathrm{~mL}$ Methyl Tert-butyl Ether (MTBE) to samples followed by vigorous vortex-mixing at room temperature. Samples were then centrifuged at $3000 \mathrm{rpm}$ for $15 \mathrm{~min}$ and the upper organic layer was then removed using a pipette and evaporated to dryness under vacuum at $45^{\circ} \mathrm{C}$ using CentriVap Console Mobile System (Labconco, USA). The dried residue was then reconstituted with $100 \mu \mathrm{L}$ of mobile phase and a $20 \mu \mathrm{L}$ aliquot was injected onto the LC-MS/MS system.

\section{Assay Validation \\ Calibration and Linearity}

Calibration curves were constructed using eight concentrations of naltrexone and its reduced metabolite (6-beta naltrexol). Each calibration level (Including blank sample with or without internal standard) was run in duplicate for three days, except for the Lower Limit of Quantification (LLOQ), which was run in 3-5 replicates. For each curve, the peak area ratios of the analyte to the internal standard were calculated and plotted against the nominal analyte concentration. Calibration curves were generated by $1 / \mathrm{x}$ weighted linear regression model that provided the best fit to data.

\section{Accuracy and Precision}

The accuracy and precision were assessed by analysis of six replicates of QC samples run on three days, with a total of $n=18$ samples at each QC level. Intra-day accuracy and precision were determined by analysing six replicates run at one day, whereas inter-day accuracy and precision were estimated using all 18 QC samples. Accuracy was expressed as (\% bias) which reflects the measured concentration relative to the nominal concentration. The precision was expressed as relative standard deviation (\% RSD). For the accuracy and precision, the acceptance criteria should be within 15\% for the QC samples and within 20\% for the LLOQ.

\section{Extraction Efficiency and Matrix Effect}

The extraction efficiency was assessed by comparing the peak areas of analytes spiked in the buffer before extraction with that of neat solutions spiked in the mobile phase, which was defined as $100 \%$ recovery. Matrix effect was evaluated by comparing the response of the analytes spiked in the buffer after sample processing with that of neat solutions spiked directly in mobile phase, representing 100\% (no matrix effect). All experiments were conducted in four replicates at the three QC levels.

\section{Application of the Method}

The current method was applied by evaluating the impact of uremic toxins on metabolic reduction using naltrexone as probe substrate. The formation of 6-beta naltrexol metabolite in vitro was quantified after naltrexone reduction. Human hepatic cytosolic protein $(0.5 \mathrm{mg} / \mathrm{mL})$ was incubated with naltrexone $(50 \mu \mathrm{M})$ and $5 \mathrm{mM} \mathrm{MgCl}_{2}$ in $10 \mathrm{mM}$ Tris- $\mathrm{HCl}$ buffer ( $\mathrm{pH}$ 7.4). Incubations were conducted in the presence of uremic toxins such as hippuric acid, p-cresol, 3-indoxyl sulfate, indole-3-acetic acid, CMPF and creatinine at previously reported concentrations of 10 and $100 \mu \mathrm{M} \cdot{ }^{14,15}$ Substrate concentration was selected based on $\mathrm{Km}$ value determined on preliminary experiments. Control incubations were performed with vehicles (DMSO or water) at a final concentration of $1 \% \mathrm{v} / \mathrm{v}$. Testosterone $(100 \mu \mathrm{M})$ that was previously shown to inhibit naltrexone reduction and was used as a positive control. ${ }^{5}$ The reaction mixture was pre-incubated for $5 \mathrm{~min}$ in a shaking water bath at $37^{\circ} \mathrm{C}$ prior to the start of the reaction by addition of $1 \mathrm{mM} \mathrm{NADPH}$. Reactions lasted for $30 \mathrm{~min}$ and terminated by the addition of ice-cold acetonitrile. Samples were then mixed briefly and placed on ice for $10 \mathrm{~min}$. The mixture was then centrifuged at 10,000 rpm for $15 \mathrm{~min}$ to pellet cytosolic protein. The supernatant was collected and the analytes were quantified as described above. Incubations were conducted in triplicates.

The formation rate (pmoles/mg protein/min) of 6-betal naltrexol metabolite reflecting the reductase enzyme activity was calculated from the measured concentrations. In the inhibition experiments, the formation rate was presented as percentage of control (vehicle) incubations. Statistical differences in the formation of metabolite were compared in the presence and absence of inhibitors using student's $t$ test assuming equal variances. The $P$ value of $<0.05$ was considered statistically significant. Analysis was performed using (GraphPad Software Inc., San Diego, CA). 


\section{RESULTS}

\section{Optimization of LC-MS/MS Conditions, Chromatography and Extraction Method}

MS parameters (tube lens and collision energy) were optimized automatically to achieve optimum detection of the analytes with best sensitivity. Individual standard solutions $(10 \mu \mathrm{g} / \mathrm{mL})$ of the analytes and internal standard were directly infused into the instrument equipped with ESI source operating in positive ionization mode. Optimum mass transitions were selected for each analyte and were used later for their quantification.

Chromatographic conditions were optimized with the aim of achieving the shortest run time and best peak shape. Various mobile phases' composition and ratios, different formic acid concentrations and variable flow rates were employed. Best peak shapes and high sensitivity were obtained when $0.1 \%$ formic acid was added to the mobile phase. The enhanced sensitivity is attributed to the better protonation of the analytes in the positive ion mode.

The sample preparation step of liquid-liquid extraction was employed to isolate the analytes from the buffer and reduce potential matrix effect associated with it. It also provides a step for preconcentration the sample if lower limit of detection is required. Multiple organic solvents (MTBE, diethyl ether, methylene chloride) were evaluated and MTBE was selected as it showed the highest extraction recovery compared to other solvents.

\section{Chromatographic Separation}

Representative chromatograms of LLOQ and study sample depicting the internal standard are displayed in Figure 1. The retention times were approximately $0.66,0.65$ and $0.88 \mathrm{~min}$ for naltrexone, 6-beta naltrexol and colchicine, respectively.

\section{Assay Validation}

The assay was found to be linear over concentration ranges of $100-8,000$ $\mathrm{ng} / \mathrm{mL}$ for naltrexone and $10-800 \mathrm{ng} / \mathrm{mL}$ for 6-beta naltrexol, with a correlation coefficient $\left(r^{2}\right)$ ranged of (0.990-0.998) for all curves. The LLOQ for each calibration curve demonstrated acceptable accuracy and precision with intra- and inter-day values of RSD and bias were within $\pm 7.3 \%$ and $\pm 11 \%$ respectively, for both analytes (Table 1 ). The signal-to-noise was greater than 10:1 for both analytes.

The intra-day and inter-day accuracy (\% bias) and precision (\% RSD) were determined at naltrexone QC concentrations of 400, 2000 and 7000 $\mathrm{ng} / \mathrm{mL}$ and 6-beta naltrexol concentrations of 40, 200 and $700 \mathrm{ng} / \mathrm{mL}$. Assay bias ranged from -14.7 to $15 \%$, while the RSD ranged from 1.5 to $13.2 \%$ for all QC levels at intand inter-day assessments (Table 1). In all cases, bias and RSD values were within $\pm 15 \%$ for all analytes.

The mean extraction efficiency of both analytes at all QC levels ranged from $73.2 \%$ to $114.8 \%$. The matrix effect was minor as the measured concentrations of both analytes were deviated by $23.8 \%$ to $37.1 \%$ from neat samples at all QC levels (Table 2). The RSD values were within $\pm 15 \%$ for all samples.

\section{Assay Application}

The current assay was successfully applied by evaluating the effect of multiple uremic toxins at 10 and $100 \mu \mathrm{M}$ on naltrexone reduction. The results revealed that indoxyl sulfate at $100 \mu \mathrm{M}$ inhibits significantly naltrexone reduction by $27 \%$ as compared to the vehicle control. Similarly, the formation rate of 6-beta naltrexol was decreased by $26 \%$ in the presence of the positive control testosterone $(100 \mu \mathrm{M})$, Figure 2 . The other investigated uremic toxins including hippuric acid, p-cresol, indole- 3-acetic acid, CMPF and creatinine did not have an effect on naltrexone reduction.

\section{DISCUSSION}

In here, we aimed at developing and validating a simple and robust LCMS/MS method for quantification of naltrexone and 6-beta naltrexol metabolite to support in vitro studies assessing phase I metabolic reduction. This assay was partially validated according to the U.S. Food and Drug Administration (FDA) guidelines for bioanalytical method validation. ${ }^{16}$ The method is simple, accurate and precise and was applied to inhibition enzyme kinetic studies of naltrexone reduction and found for the first time that indoxyl sulfate may inhibit metabolic reduction.

Carbonyl reduction is the primary Phase I metabolic pathway for xenobiotics bearing a carbonyl group. Naltrexone reduction to its primary alcohol metabolite (6-beta naltrexol) represents an example of such type of metabolism. ${ }^{3}$ A previous study characterized the reductase isoforms contributing to naltrexone reduction found that $\mathrm{AKR} 1 \mathrm{C} 1, \mathrm{AKR} 1 \mathrm{C} 2$ and AKR1C4 were able to metabolize naltrexone, with the highest efficacy was for AKR1C4. ${ }^{4}$ This suggests that the formation of 6-beta naltrexol may act as an indicative of reductase activity, which motivated the development of the current LC-MS/MS assay.

Table 1: Intra-and inter-day accuracy (\% bias) and precision (\% RSD) for LLOQ and quality control samples of analytes.

\begin{tabular}{ccccccc}
\hline \multirow{2}{*}{ Analyte } & Level & \begin{tabular}{c} 
Nominal \\
conc. \\
\cline { 5 - 7 }
\end{tabular} & & Intra-day & \multicolumn{3}{c}{ Inter-day $^{2}$} \\
\cline { 5 - 7 } Naltrexone & LLOQ & 100 & -3.8 & 5.5 & 5.4 & -5.75 \\
& LQC & 400 & 15.0 & 1.5 & 14.1 & 4.5 \\
& MQC & 2000 & 3.5 & 7.0 & 4.6 & 13.1 \\
& HQC & 7000 & 0.17 & 5.8 & 0.3 & 7.1 \\
6-beta & LLOQ & 10 & -7.3 & 6.5 & -11.0 & 9.75 \\
naltrexol & & & & & $\%$ & \\
& LQC & 40 & -14.7 & 9.2 & -1.3 & 13.2 \\
& MQC & 200 & 3.9 & 7.6 & 1.7 & 11.8 \\
& HQC & 700 & -4.6 & 4.6 & -2.1 & 4.4 \\
\hline
\end{tabular}

1 Five replicates for LLOQ; six replicates for LQC, MQC, HQC

${ }^{2}$ Eleven replicates for LLOQ; eighteen replicates for LQC, MQC, HQC

Table 2: Extraction efficiency and matrix effect of analytes in the incubation buffer.

\begin{tabular}{ccccc}
\hline Analyte & Level & $\begin{array}{c}\text { Nominal } \\
\text { concentration } \\
(\mathrm{ng} / \mathrm{mL})\end{array}$ & $\begin{array}{c}\text { Extraction } \\
\text { efficiency }(\%, \\
\text { mean } \pm \text { RSD })\end{array}$ & $\begin{array}{c}\text { Matrix effect } \\
(\%, \text { mean } \pm \\
\text { RSD })\end{array}$ \\
\hline Naltrexone & LQC & 400 & $101 \pm 3.7$ & $76.2 \pm 2.7$ \\
& MQC & 2000 & $111.9 \pm 2.6$ & $72.3 \pm 2.7$ \\
& HQC & 7000 & $114.8 \pm 3.5$ & $68.9 \pm 2.9$ \\
6-beta & LQC & 400 & $74.4 \pm 11.8$ & $62.9 \pm 15.0$ \\
& MQC & 2000 & $80.3 \pm 3.9$ & $71.9 \pm 5.4$ \\
& HQC & 7000 & $73.2 \pm 3.9$ & $71.0 \pm 1.5$ \\
\hline
\end{tabular}

RSD: relative standard deviation. 

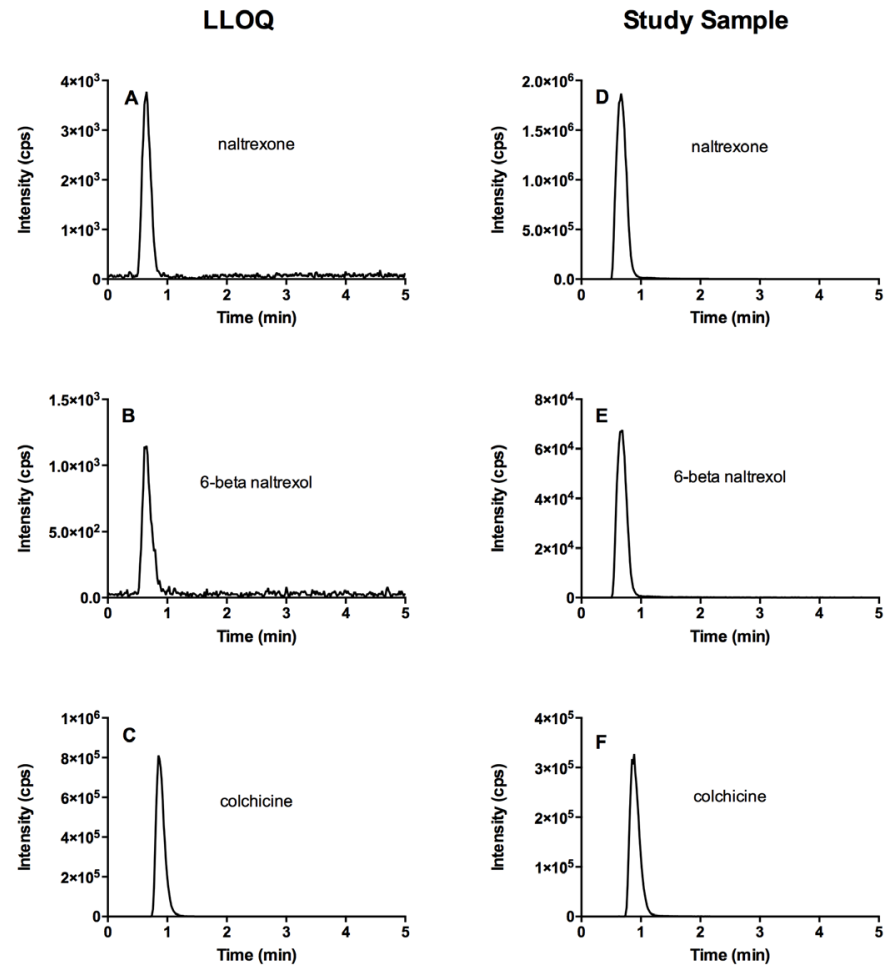

Figure. 1: Representative chromatograms of incubation buffer at LLOQ (left panel, A-C), and a cytosolic sample incubated with indoxyl sulfate (right panel, D-F). (A) naltrexone: $100 \mathrm{ng} / \mathrm{mL}$; (C, F) internal standard colchicine; (B) 6-beta naltrexol: $10 \mathrm{ng} / \mathrm{mL}$; (D) naltrexone: 7,920 ng/mL; (E) 6-beta naltrexol: $360 \mathrm{ng} /$ $\mathrm{mL}$.

While there are different analytical sensitive assays have been developed for quantification of naltrexone and its metabolites, the presence of an online sample cleaning step with column switching ${ }^{9}$ or the long run time ${ }^{10}$ may act as limitations. The current method, while it is less sensitive, it utilized a simple extraction procedure and has a short run time of 5 mins. Additionally, this assay was specifically developed to quantify 6-beta naltrexol after in vitro incubations. The previous reported methods ${ }^{11,13}$ employed a relatively large sample volume $(1 \mathrm{~mL})$ compared to our method which used $200 \mu \mathrm{L}$ sample volume. This is important, especially in enzyme kinetic studies since incubation volumes are relatively small. The linear range selected for this study is relatively wide and applicable for enzyme kinetic investigations. For instance, the concentrations of the metabolite obtained from the in vitro studies were within the calibration curve. The results of accuracy and precision experiments demonstrate that the current developed method is satisfactory valid and reproducible. Furthermore, the process efficiency was consistent and reproducible for individual analyte at the three QC levels.

Several studies have shown that kidney disease alters selectively the functional expression of drug metabolizing enzymes. ${ }^{17}$ For example, the activity of hepatic reductase enzymes that mediate metabolic reduction is found to be decreased in the setting of impaired kidney function.? However, the mechanisms behind this alteration are not well established. In here, we hypothesized that uremic toxins accumulated in the serum of kidney disease patients are implicated in this alteration. Therefore, the present method was applied by assessing the effect of various uremic toxins at 10 and $100 \mu \mathrm{M}$ on naltrexone reduction. The finding that indoxyl sulfate is an inhibitor of drug metabolism has also been reported earlier by Tsujimoto et al. who showed that metabolism of losartan in human liver microsomes mediated by CYP450 is inhibited by indoxyl sulfate in

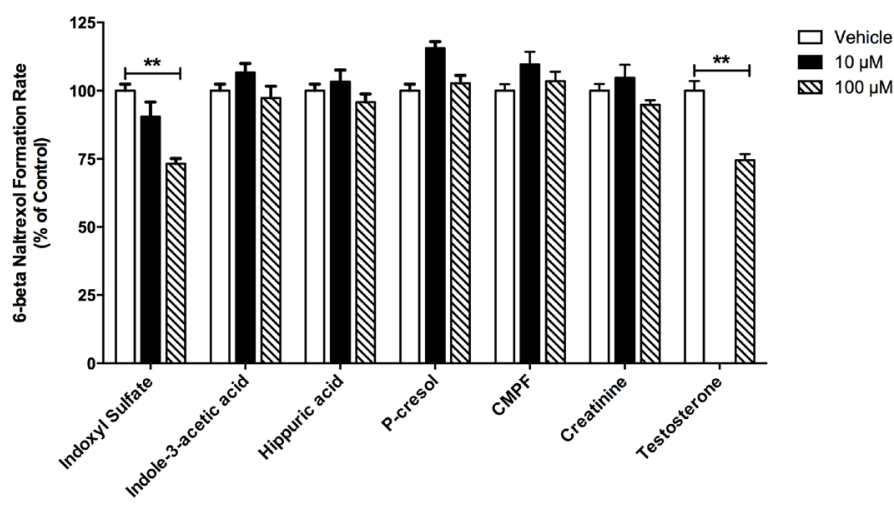

Figure. 2: Effect of uremic toxins on the metabolic reduction of naltrexone in human liver cytosol. The $y$-axis represents the formation rate of the metabolite (6-beta naltrexol) which was calculated from the measured concentrations. ** indicates $p$ value $<0.01 . n=2$ experiments.

a dose dependent manner. ${ }^{18}$ All other toxins tested in our study showed negligible effect on naltrexone metabolic reduction indicating that the effect on drug metabolism might be linked to specific uremic toxins. The latter observation is consistent in part to the results of previous studies that revealed that indole-3-acetic acid, ${ }^{14}$ creatinine, ${ }^{19} \mathrm{CMPF}$ and hippuric acid ${ }^{15}$ did not have an effect on CYP450 activity. Overall, the results indicate that the current assay is suitable for enzyme kinetics studies pertain to metabolic reduction.

The study has limitations. The sensitivity of the method was lower than previous assays. However, the range of the metabolite concentration selected was relevant to the purpose of metabolite formation in the presence of inhibitors. Also, the study did not assess the kinetic parameters of inhibitors such as $\mathrm{IC}_{50}$ or $\mathrm{Ki}$, which might be explored in future investigations.

\section{CONCLUSION}

A simple, rapid, accurate and reproducible LC-MS/MS method for the determination of naltrexone and 6-beta naltrexol metabolite has been developed and validated. These characteristics along with the short run time and the selectivity associated with LC-MS/MS analysis renders this assay well suited for conducting enzyme kinetic studies assessing metabolic reduction (i.e., naltrexone).

\section{ACKNOWLEDGEMENT}

This work was supported by the Deanship of Scientific Research at Jordan University of Science and Technology/JUST (grant number 97/2016). We gratefully acknowledge the team members of Pharmaceutical Research Center at JUST.

\section{CONFLICT OF INTEREST}

The authors declear no conflict of interest.

\section{ABBREVIATIONS}

AKR: Aldo-Keto Reductase; CMPF: 3-carboxy-4-methyl-5-propyl2-furanpropanoic acid; FDA: Food and Drug Administration; HPLC: High Performance Liquid Chromatography; HQC: High Quality Control; LLOQ: Lower Limit of Quantification; LQC: Low Quality Control; MS: Mass Spectrometry; MTBE: Methyl Tert-Butyl Ether; MQC: Middle Quality Control; QCs: Quality Control samples; RSD: Relative Standard Deviation; r2: Correlation Coefficient. 


\section{REFERENCES}

1. Hartung DM, McCarty D, Fu R, Wiest K, Chalk M, Gastfriend DR. Extended-release naltrexone for alcohol and opioid dependence:A meta-analysis of healthcare utilization studies. J Subst Abuse Treat. 2014;47(2):113-21.

2. Gonzalez JP, DenBrog RN. Naltrexone. A review of its pharmacodynamic and pharmacokinetic properties and therapeutic efficacy in the management of opioid dependence. Drugs. 1988;35(3):192-213.

3. Ohara H, Miyabe Y, Deyashiki Y, Matsuura K, Hara A. Reduction of drug ketones by dihydrodiol dehydrogenases, carbonyl reductase and aldehyde reductase of human liver. Biochem Pharmacol. 1995;50(2):221-7.

4. Breyer-Pfaff $U$, Nill K. Carbonyl reduction of naltrexone and dolasetron by oxidoreductases isolated from human liver cytosol. J Pharm Pharmacol. 2004;56(12):1601-6.

5. Porter SJ, Somogyi AA, White JM. Kinetics and inhibition of the formation of 6beta-naltrexol from naltrexone in human liver cytosol. Br J Clin Pharmacol. 2000;50(5):465-71

6. Wall ME, Brine DR, Perez-Reyes M. Metabolism and disposition of naltrexone in man after oral and intravenous administration. Drug Metab Dispos. 1981;9(4):369-75.

7. Alshogran OY, Nolin TD. Implications of Kidney Disease on Metabolic Reduction. Curr Drug Metab. 2016;17(7):663-72.

8. Brunen S, Kruger R, Finger S, Korf F Kiefer F Wiedemann K, et al. Determination of naltrexone and 6beta-naltrexol in human blood: Comparison of high-performance liquid chromatography with spectrophotometric and tandem-massspectrometric detection. Anal Bioanal Chem. 2010;396(3):1249-57.

9. Clavijo C, Bendrick-Peart J, Zhang YL, Johnson G, Gasparic A, Christians U. An automated, highly sensitive LC-MS/MS assay for the quantification of the opiate antagonist naltrexone and its major metabolite 6beta-naltrexol in dog and human plasma. J Chromatogr B Analyt Technol Biomed Life Sci. 2008;874(12):33-41.

10. Eckart K, Rohrich J, Breitmeier D, Ferner M, Laufenberg-Feldmann R, Urban $R$. Development of a new multi-analyte assay for the simultaneous detection of opioids in serum and other body fluids using liquid chromatography- tandem mass spectrometry. J Chromatogr B Analyt Technol Biomed Life Sci. 2015;1001:1-8.

11. Slawson $\mathrm{MH}$, Chen $\mathrm{M}$, Moody $\mathrm{D}$, Comer SD, Nuwayser ES, Fang WB, et al. Quantitative analysis of naltrexone and 6beta-naltrexol in human, rat and rabbit plasma by liquid chromatography-electrospray ionization tandem mass spectrometry with application to the pharmacokinetics of Depotrex in rabbits. J Anal Toxicol. 2007;31(8):453-61.

12. Valiveti $\mathrm{S}$, Nalluri BN, Hammell DC, Paudel KS, Stinchcomb AL. Development and validation of a liquid chromatography-mass spectrometry method for the quantitation of naltrexone and 6beta-naltrexol in guinea pig plasma. J Chromatogr B Analyt Technol Biomed Life Sci. 2004;810(2):259-67.

13. Yun HY, Bang SC, Lee KC, Baek IH, Lee SP, Kang W, et al. Simultaneous analysis of naltrexone and its major metabolite, 6-beta-naltrexol, in human plasma using liquid chromatography-tandem mass spectrometry: Application to a parentmetabolite kinetic model in humans. Talanta. 2007;71(4):1553-9.

14. Liu H, Narayanan R, Hoffmann M, Surapaneni S. The Uremic Toxin Indoxyl3-Sulfate Induces CYP1A2 In Primary Human Hepatocytes. Drug Metab Lett. 2016;10(3):195-9.

15. Tsujimoto M, Nagano Y, Hosoda S, Shiraishi A, Miyoshi A, Hiraoka S, et al. Effects of decreased vitamin D and accumulated uremic toxin on human CYP3A4 activity in patients with end-stage renal disease. Toxins. 2013;5(8):1475-85.

16. Dhhs US. CDER F: Guidance for Industry-Bioanalytical Method Validation. U. S. Department of Health and Human Services, Food and Drug Administration, Center for Drug Evaluation and Research, Center for Veterinary Medicine. 2018 Available: https://www.fda.gov/downloads/drugs/guidances/ucm070107.Pdf.

17. Liu B, Luo F, Luo X, Duan S, Gong Z, Peng J. Metabolic Enzyme System and Transport Pathways in Chronic Kidney Diseases. Curr Drug Metab. 2018;19(7):568-76

18. Tsujimoto M, Higuchi $K$, Shima D, Yokota H, Furukubo T, Izumi S, et al. Inhibitory effects of uraemic toxins 3-indoxyl sulfate and p-cresol on losartan metabolism in vitro. J Pharm Pharmacol. 2010;62(1):133-8.

19. Volpe DA, Tobin GA, Tavakkoli F, Dowling TC, Light PD, Parker RJ. Effect of uremic serum and uremic toxins on drug metabolism in human microsomes. Regul Toxicol Pharmacol. 2014;68(2):297-303.

Article History: Submission Date : 27-11-2018; Revised Date : 20-03-2019; Acceptance Date : 29-03-2019.

Cite this article: Alshogran OY, Zayed AL. A Simple HPLC-MS/MS Method for Quantification of Naltrexone and 6-beta Naltrexol: An Application to Effect of Uremic Toxins on Metabolic Reduction. JYoung Pharm. 2019;11(3):261-5. 